\title{
Publisher Correction: Stimulation of 3D osteogenesis by mesenchymal stem cells using a nanovibrational bioreactor
}

Penelope M. Tsimbouri, Peter G. Childs, Gabriel D. Pemberton, Jingli Yang, Vineetha Jayawarna, Wich Orapiriyakul, Karl Burgess, Cristina González-García, Gavin Blackburn, Dilip Thomas, Catalina Vallejo-Giraldo, Manus J. P. Biggs, Adam S. G. Curtis, Manuel Salmerón-Sánchez, Stuart Reid and Matthew J. Dalby

Correction to: Nature Biomedical Engineering https://doi.org/10.1038/s41551-017-0127-4; published online 12 September 2017.

In the version of this Article originally published, in Fig. 4f, the asterisk was missing; in Fig. 6a-c, the labels 'Wnt/ $\beta$-catenin signalling', 'Wnt/Ca+ pathway' and 'ERK' and their associated lines/arrows were missing; and in Fig. $6 \mathrm{~d}$ and in the sentence beginning "In MSCs that were...,' 'myosin' and 'nanostimulated', respectively, were spelt incorrectly. These errors have now been corrected in all versions of the Article. 